\title{
Un modelo de gestión cultural para entidades arqueológicas en municipios de Bolivia
}

\author{
A Cultural Management Model for Archaeological Entities \\ in the Municipalities of Bolivia
}

Um modelo de gestão cultural para entidades arqueológicas em municípios da Bolívia

\author{
Jimena Portugal Loayza \\ Universidad Mayor de San Andrés. La Paz, Bolivia \\ jpportugal@umsa.bo \\ https://orcid.org/0000-0002-7341-1850
}

DOI: https://doi.org/10.32719/25506641.2022.11.10

Recibido: 31 de marzo de 2021 • Revisado: 27 de mayo de 2021

Aceptado: 21 de junio de 2021 • Publicado: 1 de enero de 2022

Artículo de investigación 


\section{Resumen}

El objetivo es proponer un modelo de gestión para el registro, protección y planificación territorial de entidades arqueológicas para municipios en Bolivia. La investigación tiene un enfoque cualitativo, es descriptiva y propositiva, se emplea la técnica del análisis documental-bibliográfico y la experiencia de un proyecto de catastros arqueológicos municipales. Por su enfoque teórico, sigue las líneas de la cartografía cultural y la arqueología preventiva. El modelo está compuesto por el marco y plan de gestión. En el plan de gestión cultural se establecen líneas estratégicas, mecanismos de coordinación interinstitucional, gestión de talento humano y recursos financieros. Se trata de un modelo de cooperación público dependiente y cogestión, en la que participan dos instancias públicas: los municipios y el Observatorio de Patrimonio Cultural Arqueológico de la Universidad Mayor de San Andrés. Los ámbitos de aplicación del modelo de gestión son: registro, documentación, investigación, planificación territorializada y protección de las entidades arqueológicas (paisajes, sitios, hallazgos aislados). Con la aplicación del modelo de gestión se espera promover un reconocimiento del potencial del patrimonio arqueológico en los municipios. Asimismo, que el uso del catastro arqueológico municipal, como instrumento de planificación territorial, y el mejoramiento de algunos aspectos del marco normativo coadyuven en la generación de propuestas de gestión responsable y sostenible.

Palabras clave: Modelo de gestión, patrimonio cultural arqueológico, planificación territorial, cartografía cultural.

JEL: Z1 Economía de la cultura.

\section{Abstract}

The objective is to propose a management model for the registration, protection, and territorial planning of archaeological entities for the municipalities in Bolivia. The research adopts a qualitative, descriptive, and propositional approach, using the technique of documentary-bibliographic analysis and the experience of a municipal archaeological cadastre project. Due to its theoretical approach, it follows the lines of cultural cartography and preventive archeology. The model is made up of the management plan and framework. The cultural management plan establishes strategic lines, inter-institutional coordination mechanisms, and human talent and financial resources management. This is a public cooperation and co-management model, involving the participaton of two public bodies: the municipalities and the Observatory of Archaeological Cultural Heritage of Universidad Mayor de San Andrés. The areas of application of the management model are: registration, documentation, research, territorial planning, and the protection of archaeological entities (landscapes, sites, isolated findings). With the application of the management model, we expect to raise awareness of the potential of archaeological heritage in the municipalities. Similarly, the use of the municipal archaeological cadastre as a territorial planning instrument, and the improvement of some aspects of the regulatory framework will contribute to the generation of responsible and sustainable management proposals. 
Keywords: Management model, archaeological cultural heritage, territorial planning, cultural cartography.

JEL: Z1 Economy of culture.

\section{Resumo}

O objetivo é propor um modelo de gestão para o registro, proteção e planificação territorial de entidades arqueológicas para munícipios na Bolívia. A investigação tem um enfoque qualitativo, é descritiva e propositiva, emprega-se a técnica da análise documental-bibliográfica e a experiência de um projeto de cadastros arqueológicos municipais. Pelo seu enfoque teórico, segue as linhas da cartografia cultural e da arqueologia preventiva. O modelo está composto pelo marco e plano de gestão. No plano de gestão cultural se estabelecem linhas estratégicas, mecanismos de coordenação interinstitucional, gestão de talento humano e recursos financeiros. Trata-se de um modelo de cooperação pública dependente e de cogestão, na que participam duas instâncias públicas: os municípios e o Observatório de Patrimônio Cultural Arqueológico da Universidade Maior de San Andrés. Os âmbitos de aplicação do modelo de gestão são: registro, documentação, investigação, planificação territorializada e proteção das entidades arqueológicas (paisagens, sítios, recolhimentos específicos). Com a aplicação do modelo de gestão se espera promover um reconhecimento do potencial do patrimônio arqueológico nos municípios. Assim como o uso do cadastro arqueológico municipal, como instrumento de planificação territorial, e o aprimoramento de alguns aspectos do marco normativo coadjuvem na geração de propostas de gestão responsável e sustentável.

Palavras-chave: Modelo de gestão, patrimônio cultural arqueológico, planificação territorial, cartografia cultural.

JEL: Z1 Economia da cultura.

\section{Introducción}

E

1 patrimonio cultural arqueológico en Bolivia tiene un importante potencial estratégico para el desarrollo económico y social de sus habitantes; empero con una escasa consideración a su gestión sostenible. Se carece de políticas que prioricen su protección y planificación territorializada, así como de herramientas metodológicas para su registro.

Entre las causas de este problema, está una realidad común para todos los países latinoamericanos: el contexto globalizador actual produjo una desconexión de los procesos históricos y culturales, debido a la aparición de 
nuevas pautas y modelos culturales que desvalorizan e incluso ignoran el patrimonio cultural (De la Torre 2015).

Otra causa del problema es el desconocimiento de la existencia de gran parte de los sitios arqueológicos, tanto por ubicarse bajo el subsuelo, como por existir todavía muy pocos trabajos dedicados al registro sistemático de entidades arqueológicas (sitios arqueológicos, paisajes antrópicos, hallazgos aislados).

Respecto a las investigaciones arqueológicas e informes de intervenciones por proyectos de construcción de caminos, extracción minera, hidrocarburos y megaobras, además de ser guiadas por temáticas y problemáticas específicas, son de corto alcance y en espacios geográficos muy limitados.

Las reformas en la normativa del Estado han avanzado hacia un sistema descentralizado, dando a los municipios responsabilidades sobre la protección y gestión de su patrimonio arqueológico por ser la unidad territorial mínima dentro de la estructura político-administrativa. Actualmente en Bolivia se cuenta con 327 municipios (figura 1).

Los municipios tienen limitaciones en las capacidades técnicas para cumplir con estas nuevas competencias. La mayoría de los municipios no cuentan con inventarios de su patrimonio arqueológico, instrumento indispensable para un ordenamiento territorial, planificación de proyectos de desarrollo social y gestión cultural.

Ante esta problemática, se plantea como objetivo principal proponer un modelo de gestión para el registro, protección y planificación territorial de entidades arqueológicas para municipios en Bolivia. Con el fin de lograr este propósito se plantea definir el modelo de gestión, sus ámbitos de aplicación y el marco de gestión; diseñar el plan de gestión, constituido por líneas estratégicas, objetivos y actividades, $\mathrm{y}$, finalmente, formular estrategias para la gestión del talento humano y recursos financieros que permitan poner en marcha el modelo de gestión.

El modelo destaca el valor potencial de la planificación y gestión del patrimonio arqueológico que puede evitar su destrucción. La relevancia del modelo de gestión propuesto es tanto social como económica y política, pues coadyuva a la promulgación de políticas de protección y salvaguardia del patrimonio cultural. A nivel teórico-metodológico, el aporte de esta investigación es contribuir a las experiencias de la cartografía cultural, que en Bolivia no ha 
Figura 1

\section{Municipios de Bolivia}

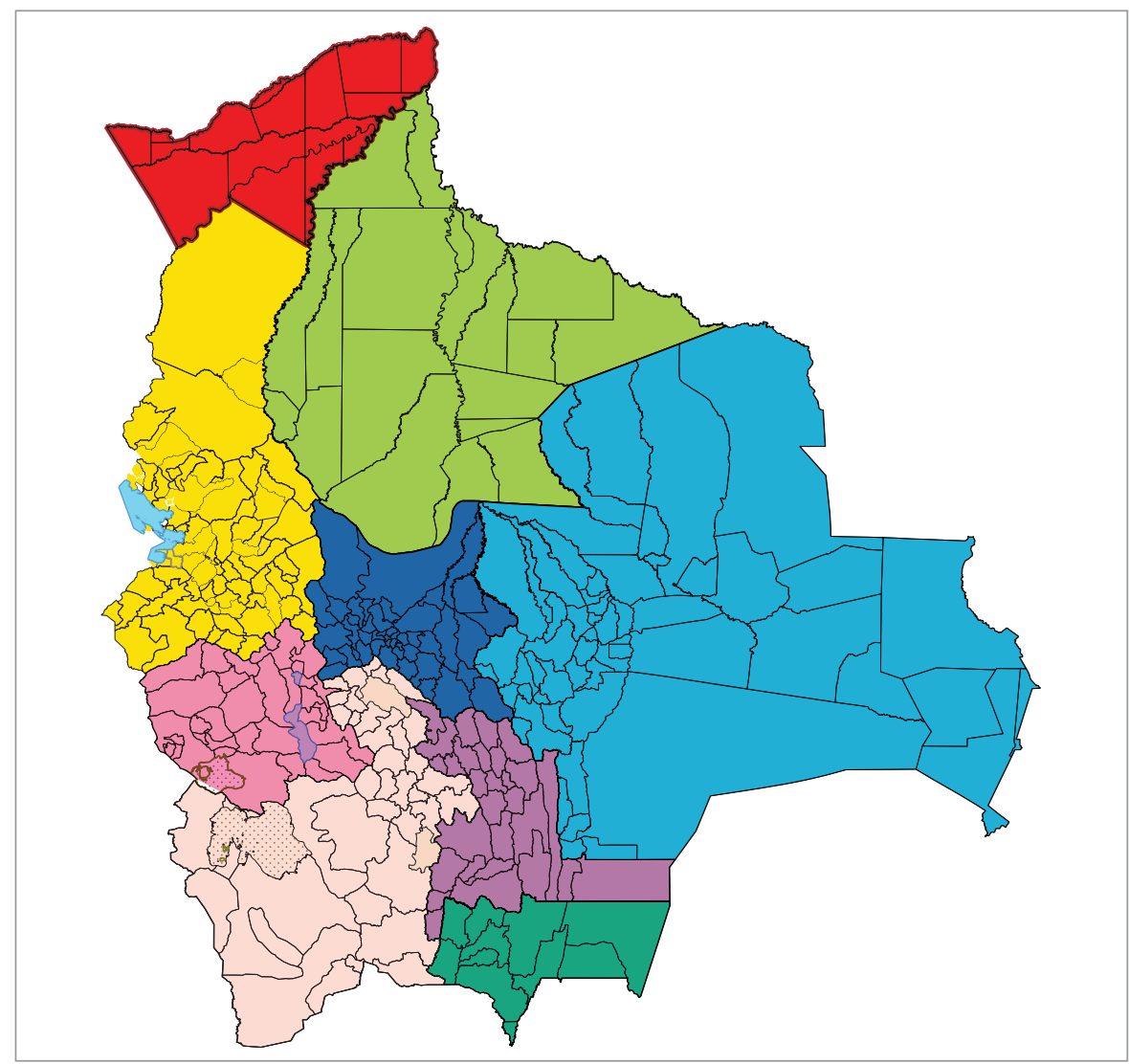

Fuente: Wikimedia Commons Contributors (2020).

sido aún visualizada como una importante estrategia en la gestión cultural y apuesta por una arqueología preventiva, en vez de limitar el ámbito de intervención en los sitios arqueológicos a una arqueología de rescate. 
El modelo, como parte de las tecnologías de la gestión, apunta al mejoramiento de resultados y procedimientos para la gestión de entidades arqueológicas; es un ejemplo de modelo colaborativo de gestión cultural.

\section{Aspectos teóricos y antecedentes}

\section{La investigación cultural contemporánea y la gestión cultural}

La gestión cultural es un campo profesional que recién empieza a tener presencia en Bolivia; se suele concretar en dos grandes subsistemas: artes y creatividad (presente-futuro), y memorias y patrimonios (presente-pasado). La gestión cultural es entendida como "aquella acción que asegura creativamente los derechos culturales, desde las artes, las memorias, y los patrimonios, y media entre las distintas culturas" (Cabrero 2013, 36). Existen tres planos en el nivel operativo del gestor cultural: nivel territorial (nacional, regional, local), nivel sectorial para promover y producir cultura, y nivel infraestructural, relacionado a las responsabilidades de gestión en una institución dada (Vives citado en Cabrero 2013).

Complementando a lo anterior, la gestión cultural es una articulación de herramientas, procesos y sentimientos para vincular a los creadores y los públicos; a los creadores con los creadores, y a los bienes patrimoniales con los públicos, desde una institucionalidad sea pública, privada o comunitaria, que permita que las construcciones estéticas y patrimoniales tengan el mejor acercamiento a la mayoría de los ciudadanos de un territorio (Cisneros 2015).

Kingman (2004) plantea que estamos viendo una formalización cultural y un vaciado de contenidos culturales del patrimonio; la tendencia de la práctica occidental es descontextualizar la alteridad y sus prácticas, neutralizar sus valores y volverlas ahistóricas. Para el trabajo del gestor cultural es esencial conocer esta realidad, ya que se toman decisiones que van más allá de decisiones técnicas y que no pueden estar desligadas de una toma de posición y considerar el contexto de manejo político en que se desarrollan las políticas culturales. 
La gestión del patrimonio cultural tiene como acciones principales: “conocer, planificar, controlar y difundir" (Querol 2017, 51). El modelo de gestión propuesto se dirige al ámbito de la planificación a nivel municipal, aunque puede ser realizado desde niveles político-administrativos mayores (gobernaciones o nivel central del Estado).

\section{Modelos de gestión cultural}

Las políticas culturales requieren de modelos de gestión para su ejecución e implementación, así también permiten a los agentes culturales actuar como sujetos de intervención en un marco normativo y democrático.

Un modelo de gestión es la "descripción del proceso administrativo que se pondrá en marcha en una organización con el fin de organizar los recursos que contribuirán a la sostenibilidad del patrimonio cultural inmueble" (Cabezas 2010, 3).

Tomando en cuenta la institucionalidad que se hace cargo de la administración y el origen de los recursos, que le permite cumplir con la finalidad, la tipología de modelos de gestión cultural se divide en: pública, privada o social. La primera está conformada por instituciones como municipios, gobernaciones y otras instituciones públicas que se hacen cargo de la administración; la privada, por instituciones como corporaciones, fundaciones, mutuales, organizaciones comunitarias, y la social es cuando un grupo de personas — sin estar constituidas legalmente - logran dar sostenibilidad a un inmueble. En cuanto al origen de los recursos, si provienen del uso del bien son modelos de gestión autónoma; si se sostienen a partir de recursos aportados por el administrador, mecenas o terceros, es un modelo de gestión dependiente, y si los recursos provienen de ambas fuentes, son modelos de gestión mixta (Cabezas 2010).

Martinell (2014) indica que los modelos de gestión se relacionan directamente con los agentes culturales, que son quienes los incentivan o plantean. Los diferentes modelos de gestión cultural están condicionados por el desarrollo de la normativa y legislación cultural, modelos de intervención del Estado, antecedentes de las políticas culturales, formas organizativas de los agentes sociales, realidad territorial, situación socioeconómica del contexto, nivel de participación y protagonismo de los agentes culturales y realidad del 
mercado cultural. La elección del modelo de gestión dependerá también de los valores y objetivos que persiga, así como de la personalidad jurídica que asuma. Los agentes culturales deben tener la capacidad de aplicar modelos de gestión acorde con el contexto y ámbito de la cultura.

La tipología de modelos de gestión, considerando el agente cultural que lo realiza y la forma en que interviene, se divide en simples y complejos de cooperación. Entre los primeros están los casos de modelos de participación de un solo agente cultural y que recibe colaboraciones, subvenciones, entre otros. Entre los modelos complejos de cooperación están: los modelos de cogestión de organizaciones con diferentes personerías jurídicas; los modelos de coproducción en relación con un proyecto u obra, y los modelos de gestión de proyectos de cooperación internacional.

\section{La cartografía cultural y gestión territorial}

El uso de la cartografía cultural resulta una herramienta básica para apoyar en la generación de información sistematizada y localizada de diferentes aspectos de la cultura, lo cual permite una identificación, diagnóstico, visibilidad y posibilidad de seguimiento de diferentes variables para la gestión cultural. La UNESCO y otras entidades que trabajan con el ámbito de la cultura, han reconocido que la cartografía cultural es una herramienta técnica crucial para preservar los elementos culturales tangibles e intangibles (Creative City Network of Canada citado en Arcila y López 2011).

La definición de cartografía cultural más aceptada, indica que se trata de un modelo de información territorial que tiene como finalidad principal la identificación y comprensión de los sistemas culturales en un área geográfica concreta con el propósito de su planificación y gestión; para ello se utilizan herramientas como los sistemas de información geográfica (SIG) e internet que ayudan a una mayor visibilidad y reconocimiento de la importancia de la cultura para el desarrollo sustentable de un territorio. Todo ese sistema se plasma en mapas temáticos y otras formas de representación que buscan formas innovadoras de comunicación (CH Consejo Nacional de la Cultura y las Artes de Chile 2001). La cartografía es el primer paso dentro del proceso de planificación cultural y se convierte en una herramienta de apoyo para 
los gobiernos locales; sirve para cartografiar el patrimonio (Arcila y López 2011), actores, actividades y bienes (Soto 2011).

¿Por qué son importantes las cartografías culturales para la gestión del patrimonio? Porque sin información confiable y mensurable es difícil apoyar la implementación y evaluación de políticas culturales, ya sean a nivel nacional, regional o local. La metodología de las cartografías culturales se ha implementado con base en las necesidades y avances tecnológicos; por otro lado, la construcción de conocimiento se ha generado desde la multidisciplinariedad. Como base se considera, desde la cartografía, el relevamiento de información in situ, que permite localizar, graficar y sistematizar los elementos culturales existentes en un territorio y propicia su síntesis y relaciones de interdependencia, que intervienen en la configuración de la realidad cultural territorial (Porro 2013).

Asimismo, la cartografía tiene la ventaja de que en el propio proceso se puede generar una concienciación de los diferentes actores sociales y fomentar su colaboración (Centre for Cultural Management 2008), pues uno de los requisitos de la cartografía cultural es su carácter participativo (Arcila y López 2011). Este será uno de los aspectos fundamentales en la metodología que propone el modelo de gestión.

Un nuevo ámbito de actuación de la tutela del patrimonio que requiere tomar mayor importancia en Bolivia es el territorio. Entre los aspectos más positivos de la patrimonialización del territorio es que contribuye a vincular, proteger y gestionar conjuntos patrimoniales en un mismo territorio, devolviéndoles su vinculación espacial e histórica, posibilitando nuevas formas de interpretación y puesta en valor, pero también la necesidad de nuevos modelos de organización y gestión (Martínez 2008).

En la gestión cultural se justifica la contextualización del territorio por la exigencia requerida en la planificación turística y políticas de desarrollo regional, enmarcadas en el aprovechamiento de los recursos existentes; asimismo, respondiendo a la demanda social de que las políticas culturales se desarrollen paralelamente a las económicas; además, la descentralización administrativa que otorga competencias a instancias político-administrativas menores al Estado (Martínez 2008). Por lo tanto, el territorio ha dejado de entenderse únicamente como el sustrato físico y/o recurso económico; más bien, desde su dimensión patrimonial es un "bien no renovable, esencial y limitado" (VV. AA. citado en Cañizares 2014, 150). 
Desde esta perspectiva, el patrimonio se entiende como herencia o legado, pero también como capacidad de actuación sobre el presente y futuro a través del talento humano, medioambiente, recursos financieros y ámbitos territoriales (Martínez 2008); se reconoce la dimensión inmaterial del patrimonio y el paisaje como "lugar de memoria" (Sabaté 2010, 11) y de integración de valores culturales y naturales (Fernández 2019). Por tanto, se supera la etapa inmovilista del patrimonio como objeto de contemplación y deleite (Álvarez citado en Martínez 2008) y donde solo está presente su dimensión material.

\section{Inventarios y catastros arqueológicos en Bolivia}

El modelo de gestión tiene como base el catastro arqueológico. El antecedente más cercano a este son las cartas arqueológicas desarrolladas en Europa y los inventarios arqueológicos realizados en diferentes partes del mundo, incluida Latinoamérica.

Si bien la Ley No. 530 del Patrimonio Cultural Boliviano (BO 2014a) establece en su art. 32 la creación del Sistema Plurinacional de Registro del Patrimonio Cultural, hasta la fecha no se ha puesto en vigencia.

El nivel central del Estado cuenta con un listado de 7000 sitios arqueológicos (Salcedo 2012), de acceso restringido para la institución. El inventario de la Gobernación Autónoma del departamento de La Paz, el siguiente nivel político-administrativo en Bolivia, cuenta con el registro de 704 sitios arqueológicos y 132 bienes inmuebles históricos, de los que se detallan datos de su ubicación, tipología, conservación y protección legal. Finalmente, otras gobernaciones no cuentan con inventarios arqueológicos.

Esta situación muestra la alta vulnerabilidad que tiene el patrimonio arqueológico boliviano en su protección causada por los limitados inventarios arqueológicos, a lo que se suma la falta de instrumentos estandarizados destinados a su gestión.

En Bolivia existen solo dos experiencias que pueden mencionarse como intentos de registrar sitios arqueológicos con fines de planificación y gestión cultural. La catalogación de sitios arqueológicos realizada entre 1983-1984 por el Instituto Nacional de Arqueología (INAR), contó con el apoyo financiero de la Organización de Estados Americanos (OEA) y se denominó catastro arqueológico. En esta primera experiencia se realizó el registro de 264 
sitios arqueológicos en provincias al norte del departamento de La Paz, sin contar con una metodología única para el catastro arqueológico, a pesar de lo cual los sitios registrados se han introducido, a través de una ficha patrón, a la base de datos del entonces Centro de Documentación del INAR (Arellano 1985). Los resultados se publicaron en la revista Arqueología Boliviana 2. Esta iniciativa no tuvo continuidad.

En 2015, el Observatorio de Patrimonio Cultural Arqueológico (OPCA), dependiente de la Universidad Mayor de San Andrés (UMSA), se presentó a un concurso de fondos financieros para investigación provenientes de los impuestos directos a los hidrocarburos (IDH), y logró obtener apoyo financiero para poner en práctica una experiencia piloto para la realización de catastros arqueológicos en dos municipios del departamento de La Paz, Jesús de Machaca y Escoma (Portugal 2017a y 2017b). En el municipio Jesús de Machaca se registraron 250 nuevas entidades arqueológicas y en el municipio de Escoma, 128. La experiencia de este proyecto fue crucial para proponer el catastro arqueológico como herramienta metodológica y componente fundamental del modelo de gestión.

La experiencia generada en los municipios de Jesús de Machaca y Escoma ha mostrado que contar con un catastro arqueológico posibilita tener un panorama general de la ubicación espacial de los sitios arqueológicos, lo que constituye la base para integrarlo en el ordenamiento territorial del municipio y para proyectos y políticas de planificación destinados a la protección, investigación y puesta en valor del patrimonio arqueológico ubicado en el territorio municipal.

Otro aspecto positivo que se destaca fue la incorporación de personas de la comunidad en los equipos de investigación, y la información directa a las autoridades locales y comunidades sobre los propósitos del catastro arqueológico y sus resultados. Se trata de un cambio importante en la investigación hacia una arqueología pública (Criado-Boado 2018; Guerrini et al. 2018); así como la incorporación de los valores y percepciones de la población local al momento de registrar e interpretar las entidades arqueológicas, lo que permitió equilibrar los valores de la memoria y científicos, además considerar las dimensiones tangibles e intangibles del patrimonio (Rogers 2019). 


\section{Metodología}

La estrategia metodológica deriva del tipo de investigación llevada a cabo, la cual es descriptiva y propositiva. Las investigaciones descriptivas buscan caracterizar y especificar las propiedades de grupos, comunidades o cualquier otro fenómeno que es sometido al análisis. Registran, miden y evalúan diversos aspectos, dimensiones y componentes de los fenómenos investigados (Batthyány y Cabrera 2011). La investigación es descriptiva porque involucra la recopilación, organización y descripción de datos, procedentes de fuentes secundarias, como de la experiencia y procedimientos procedentes de los catastros arqueológicos realizados en los municipios Jesús de Machaca y Escoma. También es propositiva porque partiendo de los resultados de la búsqueda, descripción y análisis de información, se propone un modelo de gestión para entidades arqueológicas, que puede ser aplicado en el ámbito territorial municipal.

Asimismo, el método empleado en la investigación es el cualitativo. La investigación cualitativa se concentra en el análisis de los procesos sociales, sobre todo el sentido que las personas y los colectivos dan a la acción y acerca de la construcción de la realidad social. Por su cercanía con el terreno y su preocupación por la acción, el método cualitativo apoya la práctica profesional; asimismo, la investigación cualitativa es intensiva en los casos que estudia, si bien es limitada por su muestra, pero el evento o experiencia son estudiados a profundidad (Deslauriers 2005).

Los métodos cualitativos son un modo de investigar los fenómenos sociales en el que se persiguen determinados objetivos para dar respuesta adecuada a unos problemas concretos a los que se enfrenta la investigación. $\mathrm{Su}$ objetivo es la captación y reconstrucción de significados (procesos, comportamientos, actos), se relacionan a la hermenéutica o teoría interpretativa, misma que se enfoca en la comprensión y el significado de los eventos, del comportamiento y la práctica (Little citado en Barragán et al. 2008). Del mismo modo, las investigaciones cualitativas se enfocan en temas conceptuales y normativos (Spedding 2013).

Los métodos cualitativos buscan entrar dentro del proceso de construcción social, reconstruyendo los conceptos y acciones de la situación estudiada; para ello se recurre a descripciones a profundidad, al análisis de ámbitos 
limitados de experiencia, mediante la inmersión en los contextos en los que ocurre (Ruiz 2003).

La aplicación de este método ha permitido enfocar la investigación en la resolución de un problema identificado; la falta de herramientas metodológicas de registro y planificación para entidades arqueológicas en Bolivia y la necesidad de una propuesta que resuelva limitaciones de los municipios para llevar adelante las competencias que la ley les otorga sobre el patrimonio arqueológico. El hecho de ser partícipe de la puesta en práctica de un proyecto piloto ha permitido encontrar los aspectos centrales para proponer un modelo de gestión a nivel municipal.

La principal técnica de investigación empleada es el análisis documental-bibliográfico (Tancara 1993). Según Baena (citado en Ávila 2006), la investigación documental es una técnica que consiste en la selección y compilación de información a través de la lectura y crítica de documentos y materiales bibliográficos, periódicos, centros de documentación e información.

Las etapas de la investigación han consistido en la planeación, búsqueda y colección de antecedentes e información especializada sobre el tema investigado y, posteriormente, el procesamiento de información a través de su organización y sistematización. Finalmente, la presentación argumentada de los resultados respondiendo al objetivo planteado.

\section{El modelo de gestión y ámbitos de aplicación}

Como se indicó en la introducción, el objetivo del trabajo es proponer un modelo de gestión para el registro, protección y planificación territorial de entidades arqueológicas para municipios en Bolivia.

En primera instancia es necesario definir el modelo de gestión elegido. Si se considera la institucionalidad que se hace cargo de la administración y el origen de los recursos, que le permite cumplir con la finalidad, se trata de un modelo de gestión pública dependiente (Cabezas 2010). En el modelo propuesto, la administración está a cargo de dos instituciones públicas: por una parte, los municipios, y, por otra, la UMSA, a través del OPCA; el origen 
de los recursos proviene de ambos administradores y de la gestión que hagan del financiamiento externo.

Según los agentes culturales que intervienen, corresponde a los modelos de gestión complejos de cooperación (Martinell 2014); más precisamente se trata de un modelo de cogestión entre las dos instituciones mencionadas.

En la figura 2 se esquematizan los diferentes elementos que constituyen el modelo de gestión de entidades arqueológicas. Se encuentra el ámbito territorial de su aplicación, los municipios; la cogestión, que se implementará a partir de convenios de cooperación interinstitucional entre la UMSA/OPCA y los municipios; también se encuentra el catastro arqueológico, que es la herramienta metodológica para el registro de entidades arqueológicas, y cuenta con tres componentes: una base de datos desarrollada por el OPCA, la categorización de entidades arqueológicas y la estandarización para su registro. Por razones de espacio no se detallan estos instrumentos del catastro arqueológico.

Figura 2

Modelo de gestión para entidades arqueológicas

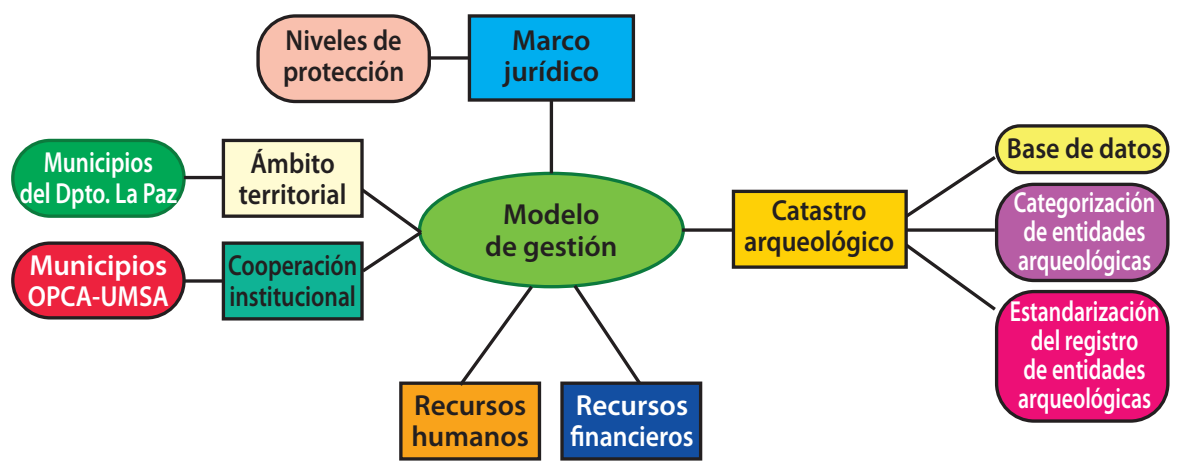

Elaboración propia. 
Según Martinell (2014), los factores que inciden e influencian los modelos de gestión son: el marco normativo jurídico y los agentes culturales condicionados por las características, dinamismos y particularidades de los distintos ámbitos de la cultura. En este caso, el agente cultural que hace la propuesta es el OPCA-UMSA, a través de una de sus investigadoras y el ámbito de la cultura corresponde al patrimonio arqueológico.

Los ámbitos de aplicación del modelo de gestión de entidades arqueológicas son su registro y documentación; investigación, que derivará en su conocimiento y difusión; la planificación territorializada, para aplicarse en el ordenamiento territorial, y la protección de las entidades arqueológicas, puesto que el modelo de gestión se deriva de la arqueología preventiva.

En la figura 3 se muestran, de forma esquemática, los ámbitos del modelo de gestión. Quedan fuera del modelo otros ámbitos, por ejemplo, la conservación, declaratorias, planes de manejo, etc., que por su especificidad requieren propuestas teórico-metodológicas particulares.

Figura 3

Ámbitos de aplicación del modelo de gestión

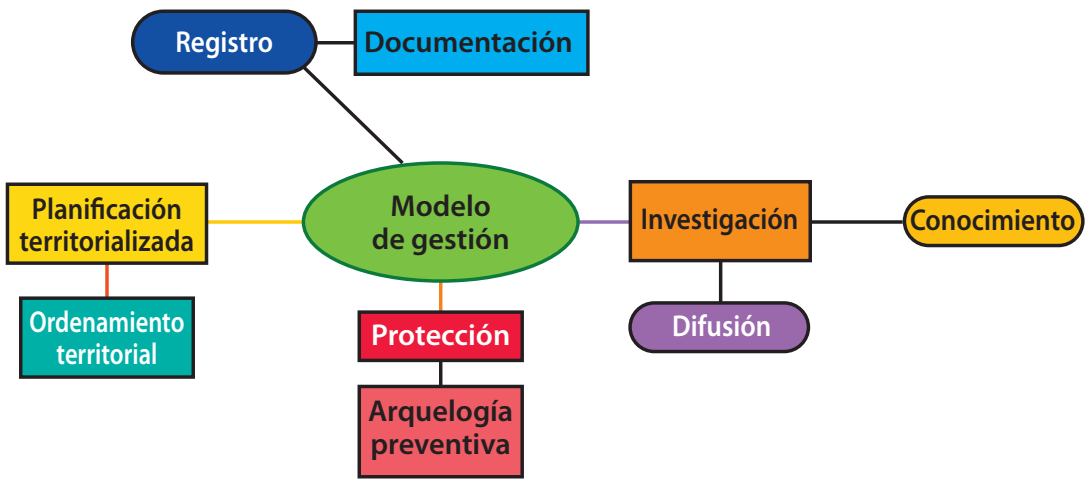

Elaboración propia. 
Según Cabezas (2010), un modelo de gestión cultural patrimonial está compuesto por el marco de gestión y el plan de gestión. Se presentan los elementos que componen el primero y el diseño del plan de gestión.

\section{El marco de gestión}

Los elementos que incluye el marco de gestión son los siguientes:

- Tipo de modelo de gestión: público dependiente-complejo de cooperación/cogestión.

- Administradores del modelo: municipios y OPCA-UMSA.

- El bien al que está dirigido el modelo: patrimonio arqueológico-entidades arqueológicas.

- Normativa jurídica: conjunto de normas y leyes referidas al patrimonio arqueológico y planificación territorial.

\section{El plan de gestión}

Dentro del plan de gestión se encuentra: la visión y los beneficios esperados con la aplicación del modelo. La visión del modelo de gestión es lograr el registro sistemático, protección, planificación territorial y gestión de las entidades arqueológicas en Bolivia, mediante la implementación de catastros arqueológicos y la cooperación interinstitucional entre el OPCA y, los municipios. Los beneficios esperados son que el conocimiento y registro de las entidades arqueológicas, el uso del catastro arqueológico municipal en la planificación territorial y el mejoramiento de algunos aspectos del marco normativo coadyuven a lograr la protección del patrimonio arqueológico y, consecuentemente, la posibilidad de generar recursos para los municipios a través de propuestas de gestión responsables y sostenibles.

Como parte del plan de gestión se encuentran las líneas estratégicas, objetivos y actividades, también los mecanismos de coordinación institucional necesarios. 


\section{Líneas estratégicas, objetivos y actividades}

Las políticas culturales requieren de líneas estratégicas para ejecutarse. Para el plan de gestión se formularon dos líneas estratégicas; los objetivos del plan de gestión se presentan con relación a cada línea estratégica y a su vez cada objetivo cuenta con un número de actividades a realizarse (tabla 1).

La concretización del modelo de gestión se logrará a partir de contar con el equipo de investigación y los recursos financieros; para ello se deben tener mecanismos de coordinación entre las instituciones responsables de ejecutarlo.

Tabla 1

Líneas estratégicas, objetivos y actividades del plan de gestión

\begin{tabular}{|c|c|c|}
\hline Líneas estratégicas & Objetivos & Actividades \\
\hline \multirow{3}{*}{$\begin{array}{l}\text { a) Registro sistemáti- } \\
\text { co y continuado de las } \\
\text { entidades arqueológi- } \\
\text { cas, para su conoci- } \\
\text { miento, investigación, } \\
\text { protección y planifi- } \\
\text { cación territorial, me- } \\
\text { diante la coordinación } \\
\text { interinstitucional. }\end{array}$} & $\begin{array}{l}\text { 1. Implementar el catastro arqueo- } \\
\text { lógico municipal en el registro de } \\
\text { entidades arqueológicas para la } \\
\text { investigación, planificación, orde- } \\
\text { namiento territorial y gestión del } \\
\text { patrimonio arqueológico en los } \\
\text { municipios. }\end{array}$ & $\begin{array}{l}\text { a) Realización de catastros arqueoló- } \\
\text { gicos. } \\
\text { b) Difundir los resultados entre la po- } \\
\text { blación local y autoridades municipa- } \\
\text { les para implementarse en la planifica- } \\
\text { ción y ordenamiento territorial. } \\
\text { c) A partir de la cartografía de entida- } \\
\text { des arqueológicas en el territorio mu- } \\
\text { nicipal, establecer áreas potenciales } \\
\text { de protección, zonas o sitios de riesgo } \\
\text { para que el municipio las incluya en la } \\
\text { gestión patrimonial. }\end{array}$ \\
\hline & $\begin{array}{l}\text { 2. Fomentar la unificación de es- } \\
\text { tándares para el registro de enti- } \\
\text { dades arqueológicas. }\end{array}$ & $\begin{array}{l}\text { a) Aplicación de los estándares de re- } \\
\text { gistro de entidades arqueológicas en los } \\
\text { catastros arqueológicos municipales. } \\
\text { b) Difundir y socializar los estándares } \\
\text { con investigadores y arqueólogos/as } \\
\text { de contrato para consensuar su apli- } \\
\text { cación a un nivel más generalizado. }\end{array}$ \\
\hline & $\begin{array}{l}\text { 3. Contar con un sistema de re- } \\
\text { gistro permanente y actualizado } \\
\text { de las entidades arqueológicas } \\
\text { para usarse en la gestión del pa- } \\
\text { trimonio arqueológico. }\end{array}$ & $\begin{array}{l}\text { a) Actualización de la base de datos } \\
\text { del OPCA con la nueva información } \\
\text { procedente de los catastros arqueoló- } \\
\text { gicos municipales. } \\
\text { b) Desarrollar protocolos de uso de la } \\
\text { base de datos el OPCA para la gestión } \\
\text { del patrimonio arqueológico municipal. }\end{array}$ \\
\hline
\end{tabular}




\begin{tabular}{|c|c|c|}
\hline \multirow{2}{*}{$\begin{array}{l}\text { b) Promover mejoras } \\
\text { en el marco jurídico y } \\
\text { políticas relacionadas } \\
\text { con el patrimonio ar- } \\
\text { queológico cultural. }\end{array}$} & $\begin{array}{l}\text { 4) Incluir en la normativa muni- } \\
\text { cipal los niveles de protección } \\
\text { referidos a entidades arqueológi- } \\
\text { cas registradas y en proceso de } \\
\text { declaración. }\end{array}$ & $\begin{array}{l}\text { a) Participación del OPCA en la socia- } \\
\text { lización de normativas y reglamenta- } \\
\text { ción sobre culturas y patrimonio. } \\
\text { b) Gestionar con los municipios la } \\
\text { creación de decretos o leyes munici- } \\
\text { pales que incorporen los dos niveles } \\
\text { de protección propuestos. }\end{array}$ \\
\hline & $\begin{array}{l}\text { 5) Impulsar una política de car- } \\
\text { tografía cultural, que priorice } \\
\text { el registro, protección y planifi- } \\
\text { cación territorial del patrimonio } \\
\text { arqueológico. }\end{array}$ & $\begin{array}{l}\text { a) Participación del OPCA en los talle- } \\
\text { res de diagnóstico y socialización de } \\
\text { los planes musnicipales de cultura. }\end{array}$ \\
\hline
\end{tabular}

Elaboración propia.

\section{Mecanismos de coordinación interinstitucional}

La mayoría de los municipios no cuentan con recursos financieros suficientes o personal técnico que se encargue de realizar los catastros arqueológicos. La Ley No. 482 de Gobiernos Autónomos Municipales de Bolivia, en su art. 5, parte II, indica que, en coordinación con organismos nacionales e internacionales competentes, los municipios precautelarán y promoverán la conservación, preservación y mantenimiento de los bienes patrimoniales arqueológicos en su jurisdicción (BO 2014b). De esta manera, esta norma posibilita la coordinación con instituciones a través de convenios y la búsqueda de financiamiento externo para lograr el registro sistemático de las entidades arqueológicas presentes en territorio municipal.

La cooperación entre el OPCA y los municipios puede lograr articular los avances tecnológicos con el registro sistemático y continuo del patrimonio arqueológico territorializado y resolver el problema de la falta de personal calificado para este trabajo en los municipios. El apoyo financiero de la cooperación internacional será fundamental para esta tarea de largo alcance. 


\section{Gestión de talento humano y recursos financieros}

Se formulan, a continuación, las estrategias que permitirán la gestión de talento humano y recursos financieros necesarios para la realización de catastros arqueológicos municipales y la concretización del modelo de gestión.

\section{Talento humano}

La realización de convenios entre el OPCA y los municipios estarían destinados a gestionar el equipo de investigación necesario para la realización de catastros arqueológicos.

La metodología de los catastros arqueológicos requiere de equipos de investigación constituidos por dos o tres personas formadas en el área de arqueología, una o dos personas del área de antropología y las autoridades comunales o comunarios que participan en el recorrido por las comunidades donde se realiza la prospección arqueológica.

Existen dos modalidades que se pueden aplicar para contar con el equipo de investigación para el catastro arqueológico:

1. Los convenios de cooperación interinstitucional permitirían a los municipios que puedan apoyar logísticamente y con un estipendio a estudiantes que realicen tesis dirigidas a realizar un catastro arqueológico en el municipio. Asimismo, la carrera de antropología/arqueología podría promover que los egresados dirijan sus tesis de licenciatura a los catastros arqueológicos municipales y conseguir becas de interacción social para fomentar estos trabajos.

El OPCA puede capacitar a los tesistas y estudiantes que realicen estas investigaciones, en los procedimientos y metodología del catastro arqueológico, así como coordinar con ellos la inclusión de la información obtenida en su base de datos y la realización de los mapas temáticos respectivos.

Si no existiesen suficientes tesistas para un catastro arqueológico, tres o cuatro estudiantes de arqueología y antropología podrían acompañar a los tesistas. También se requeriría apoyo municipal para su alimentación, hospedaje y transporte. 
2. Contratación de todo el personal técnico para el catastro arqueológico por el municipio. Esta modalidad implicaría que el municipio gestione recursos financieros externos con un aporte de contraparte, o, por el otro lado, el OPCA gestione recursos de investigación para realizar catastros arqueológicos.

En este caso, el equipo estaría conformado por un profesional arqueólogo, un profesional antropólogo, tres estudiantes de arqueología, uno de antropología y las autoridades comunales y comunarios designados por sus comunidades.

\section{Coordinación con las autoridades comunales}

Un apoyo muy importante de las autoridades y personal municipal para la realización del catastro arqueológico es el contacto con las autoridades comunales y con el equipo de investigación; es necesaria la presentación del proyecto a las autoridades originarias y sindicales de las comunidades del municipio, para contar con su participación informada y decidida.

La difusión de los valores del patrimonio arqueológico es una tarea fundamental para lograr su conservación. La defensa del patrimonio cultural inmueble y promoción de su conservación son posibles solo si la gente de las comunidades y autoridades municipales se identifica con él y conoce los valores que contiene. Tilden (citado en Gandara 2018, 44) decía: "solamente se conserva lo que se aprecia y solamente se aprecia lo que se entiende".

\section{Gestión de recursos financieros}

De los objetivos propuestos en el plan de gestión, solo la realización de los catastros arqueológicos requiere financiamiento. Los municipios tienen la capacidad de buscar socios para la realización de proyectos cofinanciados; para ello, estas instancias estatales ponen una contraparte del costo total del proyecto. La contratación de un equipo de investigación requeriría la búsqueda de financiamiento externo al municipio con la cooperación internacional; la búsqueda de financiamiento con organismos internacionales puede cogestionarse con el OPCA. 
Otra alternativa es la búsqueda de financiamiento para investigación por parte del OPCA, experiencia que ya se ha dado con los catastros arqueológicos en los municipios Jesús de Machaca y Escoma.

El aporte financiero de contraparte de los municipios corresponde a los gastos de alimentación de los tesistas y estudiantes y contratación de guías y trabajadores locales que participen de los equipos de investigación. El aporte financiero, que podría aportar la UMSA, corresponde al pago de becas de interacción social (tesistas y estudiantes), materiales para el almacenamiento de materiales arqueológicos, impresión de informes y papelería. Además, préstamo de equipos para trabajo de campo y de laboratorio.

Se debe consignar en los costos del proyecto recursos para publicación de los resultados, que tendrían que ser gestionados por los administradores (municipio y OPCA), con la posibilidad de aporte de ambas instituciones si los recursos financieros lo permiten.

\section{Conclusiones}

Numerosas son las denuncias de destrucción de sitios arqueológicos en diferentes lugares de Bolivia, ya sea por la construcción de vías o carreteras, infraestructura, proyectos de desarrollo que involucran movimiento de tierras, nuevos asentamientos poblacionales, explotación de recursos naturales, abandono, saqueo y vandalismo (Cruz 2009; Haber 2013). En Bolivia no existen aún herramientas técnicas o de planificación que coadyuven a frenar la destrucción del patrimonio cultural, por ello se ha propuesto un modelo de gestión para lograr el registro sistemático, protección y planificación territorial de las entidades arqueológicas, que pueda implementarse a nivel municipal.

Se parte por definir el tipo de modelo de gestión, ámbitos de aplicación y el marco de gestión. El modelo de gestión propuesto corresponde al tipo público dependiente, en el que intervienen dos instituciones públicas: los municipios y el OPCA de la UMSA. A su vez, se trata de un modelo de gestión compleja de cooperación, en el que las dos instituciones mencionadas cogestionan los recursos financieros y talento humano profesional-técnico. 
Respecto a sus ámbitos de aplicación, estos son registro, documentación, investigación, protección y planificación territorializada de entidades arqueológicas, en las que están comprendidos paisajes culturales, sitios arqueológicos y hallazgos aislados.

El marco de gestión puntualiza elementos como el tipo de modelo de gestión y los administradores del modelo, ya mencionados. Además, indica el bien al que está dirigido el modelo, en este caso, las entidades arqueológicas, y finalmente, la normativa jurídica en que se inscribe el modelo, que por razones de espacio no se incluyó en detalle.

Otro de los objetivos específicos fue diseñar el plan de gestión, el cual está conformado por dos líneas estratégicas: primero, el registro sistemático y continuado de las entidades arqueológicas a través de catastros arqueológicos y la promoción de mejoras en el marco jurídico y políticas culturales referidas al patrimonio arqueológico. Segundo, se plantea incluir un nivel de protección al patrimonio registrado e impulsar la cartografía cultural en la planificación territorial municipal. Se detallan los objetivos y actividades, correspondientes a cada línea estratégica; además se plantean mecanismos de coordinación interinstitucional, entre los que figura la firma de convenios de apoyo y la coordinación entre las direcciones de cultura municipales y el OPCA.

Finalizando la propuesta del modelo se formularon estrategias para la gestión del talento humano y de recursos financieros que lo pongan en marcha. Una de ellas es la coordinación interinstitucional para promover los catastros arqueológicos municipales a través de la realización de tesis de grado por estudiantes de la UMSA. También está la cogestión de recursos financieros de la cooperación internacional y programas de investigación.

Se espera que esta propuesta contribuya a promulgar políticas de salvaguarda del patrimonio arqueológico y aporte a la implementación de la cartografía cultural y la arqueología preventiva en las políticas culturales en Bolivia. Asimismo, desde las tecnologías de la gestión se quiere promover el mejoramiento de procedimientos y resultados en la planificación territorial de entidades arqueológicas. 


\section{Referencias}

Arcila, Manuel, y José Antonio López. 2011. "La cartografía cultural como instrumento para la planificación y gestión cultural. Una perspectiva geográfica”. Periférica Internacional. Revista para el Análisis de la Cultura y el Territorio 12: 15-36. https://bit.ly/2RO9wez.

Arellano, Jorge. 1985. "Presentación”. Arqueología Boliviana (2): 5.

Ávila, Héctor. 2006. Introducción a la metodología de la investigación. Chihuahua: Eumed. www.eumed.net/libros/2006c/203/.

Barragán, Rossana, Ton Salman, Virginia Ayllón, Javier Sanjinés, Erick Lange, Julio Córdova y Rafael Rojas. 2008. Guía para la formulación y ejecución de proyectos de investigación. La Paz: Programa de Investigación Estratégica en Bolivia.

Batthyány, Karina, y Mariana Cabrera. 2011. Metodología de investigación en ciencias sociales. Apuntes para un curso inicial. Montevideo: Comisión Sectorial de la Enseñanza de la Universidad de la República.

BO. 2014a. Ley No. 530 del Patrimonio Cultural Boliviano. Sucre, 23 de mayo de 2014. https://bit.ly/3bRDTaT.

---. 2014b. Ley No. 482 de Gobiernos Autónomos Municipales. La Paz, 9 de enero de 2014. https://bit.ly/3bRDTaT.

Cabezas, Claudio. 2010. Guía metodológica para la elaboración de modelos de gestión del patrimonio cultural inmueble. Santiago: Ministerio del Interior / Subsecretaría de Desarrollo Regional y Administrativo-Programa Puesta en Valor del Patrimonio.

Cabrero, Ferran. 2013. “Gestión cultural en el Ecuador”. En Memorias el II Congreso Ecuatoriano de Gestión cultural (24 al 26 de octubre de 2012). Saberes y haceres para la Gestión cultural hoy, 33-51. Cuenca: Universidad de Cuenca.

Cañizares, María del Carmen. 2014. "Paisajes culturales, ordenación del territorio y reflexiones desde la geografía en España”. Poligenos. Revista de Geografía 26: 147-180. https:// bit.ly/3fyswqT.

Centre for Cultural Management. 2008. Mapping Cultural Planning in the City of L'viv: Cultural Mapping. Amsterdam: Centre for Cultural Management y European Cultural Foundation. https://bit.ly/3rJSJFC.

Cisneros, Gabriel. 2015. "El papel del Estado en el fomento socio-cultural”. En Memorias del II y IV Congreso Ecuatoriano de Gestión Cultural. Selección de ponencias, editado por Ferran Cabrero, 189-199. Quito: Casa de la Cultura Ecuatoriana Benjamín Carrión.

Consejo Nacional de la Cultura y las Artes de Chile. 2001. Cartografía cultural de Chile. Lecturas cruzadas. Publicaciones Unidad de Estudios. Santiago: Gobierno de Chile. https:// bit.ly/3wfsB8P.

Criado-Boado, Felipe. 2018. "Ciencia pública y patrimonio: más allá de la ciencia normal y de la ciencia comunitaria”. Revista PH-IAPH 95: 102-117. https://doi. org/10.33349/2018.0.4243. 
Cruz, Sandra. 2009. "Oxtotitlán, Guerrero: la conservación de un sitio de patrimonio rupestre". En Conservación de bienes culturales: acciones y reflexiones, coordinado por Luis Fernando Guerrero, 23-43. Ciudad de México: Instituto Nacional de Antropología e Historia.

De la Torre, Francisca. 2015. "Construcción de la memoria en torno al Patrimonio". En Memorias del II y IV Congreso Ecuatoriano de Gestión Cultural. Selección de ponencias, editado por Ferran Cabrero, 137-148. Quito: Casa de la Cultura Ecuatoriana Benjamín Carrión.

Desluriers, Jean-Pierre. 2005. Investigación cualitativa. Guía práctica. Pereira: Papiro.

Fernández, Silvia. 2019. "La dimensión paisajística en la gestión del patrimonio cultural en España”. Estudios Geográficos 80 (287): 1-16. https://doi.org/10.3989/estgeogr.201943.023.

Gándara, Manuel. 2018. "De la interpretación temática a la divulgación significativa del patrimonio arqueológico". En Interpretación del patrimonio cultural. Pasos hacia una divulgación significativa en México, coordinado por Manuel Gándara y María Antonieta Jiménez, 29-96. Ciudad de México: Secretaría de Cultura-INAH. https://bit.ly/3d5UAAv.

Guerrini, Christi, Mary Majumder, Meaganne Lewellyn y Amy McGuire. 2018. "Citizen Science, Public Policy". Science 361 (6398): 134-136. https:// doi.org/10.1126/science. aar8379.

Haber, Alejandro. 2013. "Arqueología y desarrollo: anatomía de la complicidad". En Arqueología y desarrollo en América del Sur, compilado por Alexander Herrera, 11-15. Bogotá / Lima: Universidad de los Andes, Facultad Ciencias Sociales, Carrera Antropología / Instituto de Estudios Peruanos.

Kingman, Eduardo. 2004. "Patrimonio, políticas de la memoria e institucionalización de la cultura”. Revista Íconos 20: 26-34. https://doi.org/10.17141/iconos.20.2004.66.

Martinell, Alfonso. 2014. “3.4. Modelos de gestión”. En Manual Atalaya. Apoyo a la gestión cultural, coordinado y editado por Catalán Salvador y Antonio González, 1-8. Andalucía: Junta de Andalucía / Consejería de Economía y Conocimiento / Universidad de Cádiz / Universidad Internacional de Andalucía. https://bit.ly/3oUcJW.

Martínez, Celia. 2008. "Patrimonialización del territorio y territorialización del Patrimonio". Cuadernos de Arte de la Universidad de Granada (39): 251-266. https://bit.ly/3fNVSA3.

Porro, Gabriela. 2013. Cartografias culturales para el desarrollo local. En Cuarto Congreso Argentino de Cultura. Buenos Aires: Universidad Nacional Tres de Febrero. https://bit. ly/3cz8uut.

Portugal, Jimena. 2017a. Catastro arqueológico del municipio de Escoma. La Paz: UMSA-OPCA.

---. 2017b. Catastro arqueológico del municipio Jesús de Machaca. La Paz: UMSA-OPCA.

Querol, María Ángeles. 2017. Manual de gestión del patrimonio cultural. Madrid: Editorial Akal.

Rogers, Ayesha. 2019. "Values and Relationships between Tangible and Intangible Dimensions of Heritage Places". En Values in Heritage Management. Emerging Approaches and 
Research Directions, editado por Erica Avrami, Susan McDonald, Randall Mason y David Myers, 172-185. Los Angeles: The Getty Conservation Institute. https://bit.ly/3uqlM20.

Ruiz, José. 2003. Metodología de la investigación cualitativa. Serie ciencias sociales 15. Bilbao: Universidad de Deusto.

Sabaté, Joaquín. 2010. "De la preservación del patrimonio a la ordenación del paisaje. Intervenciones en paisajes culturales (Europa-Latinoamérica)". Revista Labor \& Engenho 4 (1):10-25. https://bit.ly/31AVqi4.

Salcedo, Zazanda. 2012. "Templo San Juan Bautista de Yaco: proceso de reconocimiento como patrimonio cultural del departamento de La Paz". En Memorias del 5. Foro Académico ENCRyM, 146-160. Ciudad de México: Secretaría de Cultura de los Estados Unidos Mexicanos / Instituto Nacional de Antropología e Historia y Escuela Nacional de Conservación / Restauración y Museografía "Manuel del Castillo Negrete". https://bit. ly/3bSyCje.

Soto, María Paulina. 2011. "Crónica de la cartografía cultural de Chile 14 años después". Revista Periférica para el Análisis de la Cultura y el Territorio 12: 71-79. https://bit. $1 \mathrm{y} / 3 \mathrm{vsqCgu}$.

Sppeding, Alison. 2013. "Metodologías cualitativas: ingreso al trabajo de campo y recolección de datos". En Pautas metodológicas para investigaciones cualitativas y cuantitativas en ciencias sociales y humanas, coordinado por Mario Yapu, 117-198. La Paz: Universidad para la Investigación estratégica en Bolivia U-PIEB.

Tancara, Constantino. 1993. "La investigación documental". Temas Sociales 17: 91-106. https://bit.ly/31G6NoF.

Wikimedia Commons Contributors. 2020. File: Bolivia division.png. Accedido octubre de 2020. https://bit.ly/3wNPkIT. 